Annuaire suisse de politique de développement

$17 \mid 1998$

Propriété intellectuelle : quels enjeux pour les pays en développement ?

\title{
1. Situation économique en 1996 par régions
}

\section{(2) OpenEdition}

\section{Journals}

Édition électronique

URL : http://journals.openedition.org/aspd/758

DOI : 10.4000/aspd.758

ISSN : 1663-9669

Éditeur

Institut de hautes études internationales et du développement

Édition imprimée

Date de publication : 1 avril 1998

Pagination : 127-133

ISSN : 1660-5934

Référence électronique

"1. Situation économique en 1996 par régions », Annuaire suisse de politique de développement [En ligne], 17 | 1998, mis en ligne le 30 juillet 2012, consulté le 07 septembre 2020. URL : http:// journals.openedition.org/aspd/758; DOI : https://doi.org/10.4000/aspd.758 


\section{SITUATION ÉCONOMIQUE EN 1996 PAR RÉGIONS*}

'ÉCONOMIE MONDIALE a enregistré une croissance de l'ordre de 4\% pour la troisième année consécutive en 1996 (tableau n ${ }^{\circ} 1$ ). L'activité s'est redressée dans de nombreux pays industrialisés, mais de manière différenciée selon les pays de la zone. Les pays en développement ont maintenu leur taux de croissance a plus de $6 \%$ (tableau ${ }^{\circ} 2$ ). Les pays d'Amérique latine qui avaient été touchés par la crise financière mexicaine de 1995 ont renoué avec l'expansion. La croissance économique s'est aussi accélérée au Moyen-Orient et, de manière plus marquée, en Afrique. Dans les pays en transition la production s'est globalement stabilisée après six années de fortes contractions. Enfin une modération de la croissance a été notée dans plusieurs des économies émergentes d'Asie (tableau $\left.\mathrm{n}^{\circ} 3\right)^{1}$.

Tableau n ${ }^{\circ} 1$

Taux de croissance annuel du produit intérieur brut

(en pour-cent)

\begin{tabular}{lrrrr}
\hline & $\mathbf{1 9 9 3}$ & $\mathbf{1 9 9 4}$ & $\mathbf{1 9 9 5}$ & $\mathbf{1 9 9 6}$ \\
\hline Monde & $\mathbf{2 . 7}$ & $\mathbf{4 . 1}$ & $\mathbf{3 . 7}$ & $\mathbf{4 . 0}$ \\
\hline Pays industrialisés & 1.2 & 3.1 & 2.5 & 2.5 \\
\hline Pays en voie de développement $^{\prime}$ & 6.5 & 6.8 & 6.0 & 6.5 \\
\hline Pays en transition $^{\prime}$ & -6.3 & -6.7 & -0.8 & -0.1 \\
\hline
\end{tabular}

1. Ex-URSS, Bulgarie, Tchéquie, Slovaquie, Roumanie, Pologne, Hongrie.

Source: FMI, Rapport annuel 1997

\subsection{PAYS INDUSTRIALISÉS ${ }^{2}$}

La croissance a atteint 2,5\% en 1996 dans les pays de l'OCDE, soit un taux à peu près identique à celui de 1995, mais son évolution a été assez inégale. Les plus fortes impulsions ont découlé des économies américaine et britannique. La demande intérieure, dans ces deux pays, en particulier la consommation, a progressé, mais les exportations ont évolué de manière inégale. La fermeté du dollar et la baisse de la demande en Europe ont freiné les exportations américaines, alors que les ventes à l'étranger du Royaume-Uni ont encore augmenté.

* Ce chapitre a pour objectif de brosser un tableau succinct de la situation économique en 1996 par grandes régions géographiques et de replacer les relations entre la Suisse et les pays en développement du Sud et d'Europe centrale et orientale dans le contexte de l'évolution économique de ces pays. Les données fournies dans le présent chapitre proviennent des rapports annuels des principales organisations économiques (Fonds monétaire international, Banque mondiale, Banque des règlements internationaux, Organisation de coopération et de développement économiques).

1. Fonds monétaire international, Rapport annuel 1997, Washington, FMI, avril 1997, p. 11.

2. Banque nationale suisse, $89^{\circ}$ rapport de gestion 1996, Zurich, BNS, 1997, p. 7. 
Au second semestre, le continent européen a commencé à surmonter la phase de faiblesse qui le caractérisait depuis l'automne 1995, grâce à une demande étrangère plus ferme (France et Allemagne).

Après avoir stagné pendant plusieurs années, le produit intérieur brut du Japon a nettement augmenté en 1996. Sa progression s'explique surtout par les gros investissements des collectivités publiques. En dépit de taux d'intérêt bas, la demande du secteur privé n'a pas vraiment décollé, en partie sans doute à cause de la crise que subit le système bancaire japonais. La croissance des exportations a presque cessé sous l'effet du très fort raffermissement du yen entre le début de 1993 et le milieu de 1995. Quant aux importations, elles ont augmenté vigoureusement.

La solide croissance économique aux Etats-Unis et au Royaume-Uni a engendré une amélioration de l'emploi et un repli du chômage. Par contre le chômage s'est accru dans de nombreux pays européens, notamment en Allemagne et en France. En moyenne des pays de l'UE, le taux de chômage est resté inchangé à environ $11 \%$.

\section{$\square$ Economie suisse $^{3}$}

Contrairement aux prévisions énoncées en 1995, l'économie suisse a une fois encore subi une légère récession en 1996. A l'exclusion d'une courte reprise en 1994, l'économie helvétique se trouve dans une phase de stagnation pour la sixième année consécutive. Diverses raisons expliquent cette situation, presque unique dans les pays industrialisés occidentaux. Premier constat, la forte appréciation du franc suisse, depuis 1992 et qui s'est prolongée jusqu'à mi-1996, a joué un rôle primordial. En outre, l'économie suisse se trouve dans une intensive phase d'adaptation dans un contexte de globalisation de l'économie. La Suisse doit s'adapter à une division internationale du travail toujours plus forte, ainsi qu'à une concurrence mondiale accrue entre les divers sites de production. Elle doit également gérer une surcapacité dans la construction et surtout des déficits structurels des finances publiques. Ces différents éléments ne sont pas sans avoir des répercussions sur l'emploi (croissance massive et partiellement irréversible du chômage) et sur la consommation des ménages (détérioration de la consommation sans précédent, tant par son ampleur que par sa persistance). La principale composante de la demande intérieure, à savoir la consommation des ménages, est ainsi sérieusement contrariée. Les investissements des entreprises, qui avaient fortement augmenté en 1994 et 1995, continuent de se limiter aux efforts visant à maintenir la compétitivité des structures de production existantes.

En conséquence, seules des impulsions positives de l'économie extérieure peuvent être attendues. Or, en 1996, on a enregistré une contribution faiblement négative ou, dans le meilleur des cas, neutre de l'économie extérieure. Les exportations suisses ont souffert d'une conjoncture très modérée en Europe occidentale, qui compte ses principaux clients, ainsi que des répercussions de la hausse du franc qui a généré d'importantes pertes de parts de marché à l'étranger et dans certains secteurs de l'économie, tel le tourisme. Au vu de la force du franc suisse, certaines industries d'exportation, principalement les produits pharmaceutiques, les biens d'investissement et les denrées alimentaires, ont enregistré de bonnes

3. «Rapport sur la politique économique extérieure $96 / 1+2 », 15$ janvier 1997, message 97.002, pp. 28 et suivantes. 
performances avec une augmentation du volume des exportations de $2,4 \%$ en 1996.

En 1997, la situation économique s'est un peu améliorée. Selon les statistiques de l'Office fédéral des questions conjoncturelles (OFQC) publiées en décembre 1997, le produit intérieur brut a progressé de $1,7 \%$ en termes réels au troisième trimestre par rapport au second. En 1997, la croissance se chiffre à $0,8 \%$. L'OFQC reconnaît que le rythme reste modéré, mais souligne que c'est la première fois depuis deux ans que le PIB a atteint un tel taux de croissance trimestriel sur douze mois. Dopées par un cours du franc suisse nettement plus favorable qu'en 1995-1996 et par la reprise de la demande étrangère en Europe, les exportations helvétiques sont indéniablement le moteur principal de la reprise ${ }^{4}$.

\subsection{PAYS EN DÉVELOPPEMENT}

La croissance économique est restée vigoureuse et l'inflation a encore diminué, l'an dernier, dans les économies émergentes (tableau n ${ }^{\circ} 3$ ). En Amérique latine, le taux de croissance moyen a augmenté, grâce à la reprise en Argentine et au Mexique. L'Afrique a connu sa plus forte expansion depuis deux décennies, résultat des efforts de stabilisation et de réforme (selon le FMI), d'une amélioration des cours des matières premières et d'une augmentation des investissements. En Asie, plusieurs pays ont adopté des politiques restrictives pour faire face à un début de surchauffe. L'inertie des marchés d'exportation a contribué au ralentissement de l'activité dans cette région par rapport à son dynamisme antérieur et a empêché de réduire sensiblement les importants déséquilibres extérieurs. Dans un certain nombre de pays d'Europe orientale où le processus de transition est avancé, la croissance est restée soutenue. En Russie, toutefois, le redémarrage de la production doit encore se confirmer, mais la baisse tendancielle de l'inflation s'est poursuivie.

\section{Tableau n ${ }^{\circ} 2$}

\section{Taux de croissance du PNB annuel des pays en voie de développement}

\begin{tabular}{lrrrr}
\hline & $\mathbf{1 9 9 3}$ & $\mathbf{1 9 9 4}$ & $\mathbf{1 9 9 5}$ & $\mathbf{1 9 9 6}$ \\
\hline Ensemble & $\mathbf{6 . 5}$ & $\mathbf{6 . 8}$ & $\mathbf{6 . 0}$ & $\mathbf{6 . 5}$ \\
\hline Afrique & 0.9 & 2.9 & 2.9 & 5.1 \\
\hline Asie & 9.3 & 9.6 & 8.9 & 8.2 \\
\hline Moyen-Orient $^{\prime}$ & 4.3 & 0.3 & 3.8 & 4.5 \\
\hline Amérique latine et Caraïbes & 3.7 & 5.0 & 1.3 & 3.5 \\
\hline
\end{tabular}

Remarque: les chiffres de la croissance par grandes régions ne sont pas les mêmes que ceux du rapport BRI, ce qui s'explique par les différentes méthodes statistiques utilisées par les institutions financières.

1. Y compris Chypre, Malte et la Turquie.

Source: FMI, Rapport annuel 1997.

4. Journal de Genève, 11 décembre 1997 «Dopée par les exportations, mais pénalisée par la construction, l'économie suisse croît modérément». 
Sous l'effet de politiques monétaires restrictives et d'une décélération de la croissance des exportations, la croissance du volume de la production des économies dynamiques d'Asie ${ }^{5}$ (EDA) s'est quelque peu ralentie en 1996 où elle est estimée à $6 \%$. La décélération des exportations a été due pour partie à la faiblesse de la demande dans la zone de l'OCDE et sur les marchés régionaux et pour partie aux effets négatifs sur la compétitivité de l'appréciation du dollar, auquel beaucoup de monnaies de la région sont liées. Parallèlement l'inflation a très légèrement diminué pour s'établir à 4,5\%. Le ralentissement de l'activité a été particulièrement prononcé en Thaillande, où la demande intérieure a notamment souffert des effets sur les bilans, et la confiance, de la baisse des marchés boursiers immobiliers. A Taiwan et à Hongkong la décélération a été relativement moins prononcée, grâce notamment à la croissance soutenue de la Chine.

En Chine, les pressions de la demande se sont encore atténuées. La politique restrictive et le contrôle rigoureux des nouveaux projets d'investissement des entreprises publiques ont largement contribué à cette évolution. Le ralentissement sensible de la croissance des exportations, qui est revenue de près de $25 \%$, en dollars, en 1995 à moins de 5\% en 1996, a aussi joué un rôle modérateur. La hausse des prix de détail est tombée d'un taux annuel de $15 \%$ en 1995 à $6 \%$ en 1996. La croissance du PIB réel s'est réduite légèrement à $10 \%$, la relativement bonne performance du secteur agricole ayant empêché une décélération plus prononcée de la production globale. Depuis la fin de 1996, la croissance des exportations s'est sensiblement accélérée. Les exportations vers les Etats-Unis, qui représentent près de $18 \%$ des exportations chinoises totales, ont été particulièrement vigoureuses ${ }^{6}$.

En Inde, les progrès accomplis depuis 1991 dans l'ouverture des échanges commerciaux, la libéralisation du secteur financier et la réforme budgétaire ont contribué à l'accélération de la croissance, qui a atteint $6 \%$ en moyenne au cours des cinq dernières années. Dans le même temps, l'inflation a été réduite?

U ASTM 1998, «Relations financières internationales», p. 135.

\section{$\square$ Amérique latine}

Le taux de croissance de l'Amérique latine a passé de $0,5 \%$ en 1995 à 3,5\% en 1996. L'augmentation du taux de croissance moyen s'explique principalement par la reprise en Argentine et au Mexique. Parallèlement différentes stratégies de lutte contre l'inflation ont porté leurs fruits ces dernières années: le taux moyen de l'inflation se situe à $25 \%$ en 1996 . Il était de $43 \%$ en 1995, ce qui correspondait déjà à un bon résultat, puisque pendant la période 1990-1997 le taux d'inflation moyen était de $257 \%$.

Le redressement de l'activité économique, qui s'est amorcé au milieu de 1996, continue de se renforcer, surtout en Argentine, au Brésil et au Pérou. Dans ces trois pays, l'accroissement des entrées de capitaux et la détente des taux d'intérêt nominaux intérieurs ont créé les conditions d'une reprise généralisée; la

5. Taiwan, Hongkong, Malaisie, Philippines, Singapour et Thaïlande.

6. OCDE, Perspectives économiques de l'OCDE 1997, Paris, OCDE, juin 1997, pp. 133-134.

7. Banque des règlements internationaux, 67 rapport annuel, Bâle, BRI, juin 1997, pp. 47.

8. Banque des règlements internationaux, op. cit. 
Tableau n 3

Croissance et inflation

PIB réel

Prix à la consommation

\begin{tabular}{|c|c|c|c|c|c|c|}
\hline \multirow[t]{2}{*}{ Pays/Région } & \multicolumn{5}{|c|}{ Variation annuelle en pour-cent } & \multirow[b]{2}{*}{1996} \\
\hline & $1990-94$ & 1995 & 1996 & $1990-94$ & 1995 & \\
\hline & moyenne & & & moyenne & & \\
\hline Chine & 10.5 & 10.2 & 9.7 & 11.6 & 16.8 & 8.3 \\
\hline Inde & 4.6 & 7.1 & 6.8 & $10.5^{1}$ & $9.3^{1}$ & $5.9^{\prime}$ \\
\hline Autres pays d'Asie $^{2}$ & 7.0 & 7.8 & 6.8 & 6.9 & 6.4 & 5.9 \\
\hline Corée & 7.6 & 8.9 & 7.1 & 7.0 & 4.5 & 5.0 \\
\hline Hongkong & 5.2 & 4.7 & 4.7 & 9.5 & 9.2 & 6.3 \\
\hline Singapour & 8.6 & 8.8 & 7.0 & 2.9 & 1.7 & 1.4 \\
\hline Taiwan & 6.5 & 6.1 & 5.7 & 3.8 & 3.7 & 3.1 \\
\hline Indonésie & 6.9 & 8.5 & 7.5 & 8.6 & 9.4 & 7.9 \\
\hline Malaisie & 8.7 & 9.5 & 8.2 & 3.8 & 3.4 & 3.5 \\
\hline Philippines & 1.9 & 4.8 & 5.5 & 11.6 & 8.1 & 8.4 \\
\hline Thaïlande & 9.0 & 8.6 & 6.7 & 4.8 & 5.8 & 5.8 \\
\hline Amérique latine ${ }^{2}$ & 3.1 & 0.5 & 3.5 & 256.8 & 42.5 & 24.7 \\
\hline Argentine & 6.8 & -4.6 & 4.4 & 148.3 & 3.4 & 0.2 \\
\hline Brésil & 0.9 & 4.2 & 2.9 & 1425.9 & 66.0 & 15.5 \\
\hline Chili & 6.4 & 8.5 & 7.2 & 17.4 & 8.2 & 7.4 \\
\hline Colombie & 4.3 & 5.2 & 2.1 & 26.3 & 20.9 & 20.9 \\
\hline Mexique & 3.9 & -6.2 & 5.1 & 16.1 & 35.0 & 34.4 \\
\hline Venezuela & 3.9 & 3.4 & -1.6 & 40.7 & 59.9 & 99.9 \\
\hline Europe orientale $^{2}$ & -2.5 & 5.5 & 4.7 & 62.1 & 23.2 & 17.8 \\
\hline Hongrie & -3.3 & 1.5 & 1.0 & 25.4 & 28.3 & 23.6 \\
\hline Pologne & -1.6 & 7.0 & 6.0 & 97.6 & 27.7 & 19.9 \\
\hline République tchèque & -4.2 & 4.8 & 4.4 & 20.5 & 9.1 & 8.8 \\
\hline Fédération de Russie & -8.7 & -4.2 & -6.0 & 347.0 & 197.5 & 47.7 \\
\hline Arabie Saoudite & 4.1 & 1.6 & 2.4 & 1.7 & 4.9 & 1.2 \\
\hline Israël & 5.8 & 7.1 & 4.4 & 14.2 & 10.0 & 11.3 \\
\hline Afrique & 1.4 & 2.7 & 5.1 & 37.2 & 38.4 & 25.0 \\
\hline Afrique du Sud & 0.1 & 3.3 & 3.1 & 12.4 & 8.6 & 7.4 \\
\hline
\end{tabular}

Remarque: les données pour 1996 sont en partie estimées.

1. Prix de gros.

2. Moyenne pondérée des pays cités, sur la base des PIB et PPA (parités pouvoirs d'achats) de 1990.

Source: Banque des règlements internationaux, 67` Rapport annuel, juin 1997. 
croissance de la consommation privée et de l'investissement fixe a été particulière-ment soutenue. Un secteur pétrolier en pleine expansion a également suscité une reprise au Venezuela, après la contraction brutale de la production liée au plan de stabilisation du début de 1996. Sauf en Argentine, l'inflation reste forte, maiscontinue de décroître à la faveur de taux d'intérêt réels à court terme élevés, de niveaux de chômage importants et d'une légère appréciation du taux de change réel. Au Chili et en Colombie, l'activité économique s'est ralentie en 1996 du fait de la rigueur des politiques monétaires. L'inflation au Chili s'inscrit de nouveau dans une tendance à la baisse, ce qui a permis l'amorce d'une réduction progressive des taux d'intérêt. Les déficits courants se sont encore creusés dans la plus grande partie de la région en 1996, car le service de la dette étrangère s'est alourdi; la demande d'importations s'est accélérée et les termes de l'échange se sont dégradés dans la plupart des pays à l'exception du Venezuela9.

\section{$\square$ Afrique}

En Afrique, le redressement économique amorcé en 1994 et 1995, s'est encore amplifié en 1996 (tableaux $n^{\text {os }} 2$ et 3). L'expansion de 5\% observée en 1996 constitue en fait la meilleure performance de l'Afrique ces vingt dernières années. De plus, pour la première fois, le taux de croissance a été supérieur à l'augmentation de l'accroissement démographique. Cette accélération de la croissance témoigne du dynamisme du secteur des produits primaires et, parfois, du secteur manufacturier. Les pays de la zone franc ont connu une reprise soutenue après le réalignement du franc CFA en 1994. L'Ethiopie, le Malawi, le Maroc et la Tunisie figurent aussi parmi les pays où l'expansion a été vigoureuse. Par contre, la réduction indispensable des déséquilibres macro-économiques et les rigidités structurelles ont continué à freiner la croissance de l'Afrique du Sud $^{10}$. La CNUCED relève que la vaste reprise observée en Afrique subsaharienne est due principalement à une meilleure gestion des affaires publiques et à la fermeté des prix de certains produits de base, ainsi qu'à des conditions météorologiques nettement plus clémentes et à un recul des troubles civils" ${ }^{11}$.

\subsection{PAYS EN TRANSITION}

La transition se poursuit dans les économies d'Europe centrale et orientale. La fragilité de la croissance est cependant devenue manifeste, et dans certains cas préoccupante. De nombreux Etats de la région sont largement tributaires de l'expansion des exportations et ont donc souffert en 1996 du ralentissement intervenu en Europe occidentale. Des problèmes résident également dans l'offre, qui reflète des difficultés rencontrées pour améliorer la qualité et la diversité des produits. Les taux de chômage ont continué de diminuer, mais la lenteur de ce recul traduit des déficiences structurelles, notamment des disparités régionales. En Russie, on a pu observer une forte réduction du taux d'inflation, ce qui devrait permettre à la production de redémarrer. En Pologne, où la phase d'expansion

9. OCDE, Perspectives économiques de l'OCDE 1997, Paris, OCDE, juin 1997, p. 136.

10. Fonds monétaire international, Rapport annuel 1997, Washington, FMI, avril 1997, p. 14.

11. Conférence des Nations Unies sur le commerce et le développement, Rapport sur le commerce et le développement 1997, Genève, CNUCED, 1997. 
entrait dans sa cinquième année, la croissance s'est un peu modérée pour avoisiner les 5,5\%. La croissance a ralenti en Hongrie pour s'établir à $1 \%$. Par contre l'évolution observée en Bulgarie et en Roumanie montre que les politiques de transition ne permettent pas une transformation structurelle suffisante, ce qui, selon l'OCDE, peut nuire à la crédibilité des politiques monétaires et économiques globales. Les perspectives semblent généralement plus satisfaisantes en matière de croissance économique et de flux d'échanges pour 1997, mais la restructuration de l'économie planifiée vers l'économie de marché demeure complexe à mettre en œuvre ${ }^{12}$.

\section{SOURCES}

«Rapport sur la politique économique extérieure 96/1 + 2», 15 janvier 1997, message 97.002

Banque des règlements internationaux, 67' rapport annuel, Bâle, BRI, juin 1997.

OCDE, Perspectives économiques de l'OCDE 1997, Paris, OCDE, juin 1997.

Fonds monétaire international, Rapport annuel 1997, Washington, FMI, avril 1997.

Banque nationale suisse, 89' rapport de gestion 1996, Zurich, BNS, 1997.

Banque mondiale, L'Etat dans un monde en mutation - Rapport sur le développement dans le monde 1997, Washington, Banque mondiale, juin 1997.

United Nations Conference on Trade and Development, Trade and Development Report 1997, New York and Geneva, UNCTAD, 1997.

United Nations Conference on Trade and Development, The Least Developed Countries 1997 Report, New York and Geneva: UNCTAD, 1997.

\section{SITES INTERNET}

Banque mondiale: http://www. worldbank. org/

Fonds monétaire international: http://www. imf. org/

OCDE: http://www. OECD. ORG/

CNUCED: $h$ ttp://www. unicc. org/unctad/fr/frhome. htm

12. OCDE, Perspectives économiques de l'OCDE 1997, Paris, OCDE, juin 1997, p. 129. 